\title{
Monitoring ultrashort pulses by transverse frequency doubling of counterpropagating pulses in random media
}

\author{
Robert Fischer, ${ }^{\text {a) }}$ Dragomir N. Neshev, Solomon M. Saltiel, ${ }^{\text {b) }}$ Andrey A. Sukhorukov, \\ Wieslaw Krolikowski, and Yuri S. Kivshar \\ Nonliner Physics Centre and Laser Physics Centre, Centre for Ultrahigh-bandwidth Devices for Optical \\ Systems (CUDOS), Research School of Physical Sciences and Engineering, Australian National \\ University, Canberra, Australian Capital Territory 0200, Australia
}

(Received 1 May 2007; accepted 30 May 2007; published online 19 July 2007)

\begin{abstract}
The authors study experimentally the transverse second-harmonic generation of counterpropagating pulses by a quasi-phase-matching in a medium with a random ferroelectric domain structure. The authors show that this parametric process results in a direct realization of the cross correlation of two optical signals and, therefore, it can be employed for direct characterizations of ultrashort pulses including their temporal structure and pulse front tilt. () 2007 American Institute of Physics.
\end{abstract}

[DOI: $10.1063 / 1.2751583$ ]

The use of ultrashort optical pulses has become an essential tool in a rapidly increasing number of applications in both research and industry. While in most cases a precise knowledge of the pulse duration is essential for the success of such applications, characterization of pico- and femtosecond pulses requires elaborate, alignment-critical tools with usually large footprint. In this letter we demonstrate a simple method to measure pulse auto- and cross correlations in disordered media. The correlation traces result from quasiphase-matching (QPM) the process of transverse secondharmonic generation (SHG) of two counter propagating pulses in a medium with a random ferroelectric domain structure. This method directly translates time into space coordinates and thus allows for not only straightforward monitoring of the pulse duration and its front tilt but also timing information such as delay, synchronization, and jitter.

Usual pulse-characterization techniques such as standard autocorrelators, ${ }^{1}$ FROG, ${ }^{2}$ or SPIDER $^{3}$ are based on the analysis of the second harmonic (SH) generated by two pulses copropagating collinearly or under a small angle through a nonlinear material. They rely on series of interferometric measurements with an accurately varied time delay between the two pulses, thus requiring accurate and time consuming alignment. Improved techniques such as GRENOUILLE ${ }^{4}$ overcome most of these problems but they assume the optical signal to consist of only a single repeated pulse, while information on a time scale larger than the pulse length, e.g., that coming from a delayed pulse echo, is lost.

On the other hand, autocorrelation measurements based on the two-photon fluorescence (TPF) of counterpropagating pulses in dyes provide simplicity and a far wider time window require no variable delay lines. Although the autocorrelation traces do not permit retrieving of complex pulse temporal information (pulse profile and chirp), this method allows for single-shot pulse duration estimation. Variations of the TPF technique were a standard tool in the early days of mode-locked lasers. ${ }^{5,6}$ Despite their advantages, the TPF method has lost its popularity due to the high peak power requirement (about $\left.2-20 \mathrm{GW} / \mathrm{cm}^{2}\right),{ }^{6,7}$ and fast dye degradation. Extension of this method to parametric processes in

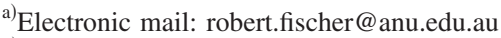

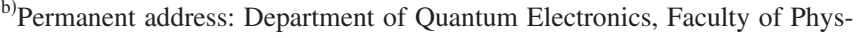
ics, Sofia University, 1164 Sofia, Bulgaria.
}

solid-state materials is not trivial since phase matching the SHG process of counterpropagating beams by birefringence is physically impossible. An option is to employ a planar waveguide geometry leading to the so-called surfaceemitting SHG. However, the efficiency of this process in the non-phase-matching regime is low. ${ }^{8-10}$ The efficiency can be enhanced by the use of transverse phase matching. In order to phase match a process where one photon from each counterpropagating pulse contributes to the generation of a single photon at the sum frequency, one needs to design a periodic photonic structure with reciprocal wave-vector $\mathbf{g}$ matching the $\mathbf{k}$ vector of the generated photon. ${ }^{10}$ This imposes a fundamental constrain on practical realization of devices as the required periodicity is of a subwavelength scale. In the past there have been several attempts to realize such nanoscale photonic structures. ${ }^{11-13}$ However, in all those cases the device performance has been limited to a narrow frequency range, which, together with difficulties in fabrication, limits its practical applicability.

To overcome this problem we propose to employ the process of parametric upconversion in media with randomly oriented ferroelectric domains. Such random media are known to quasi-phase-match any parametric process, e.g., SHG or sum-frequency mixing in an ultrabroad frequency range. ${ }^{14}$ An example of such a medium is the naturally grown strontium barium niobate (SBN) crystal. Without the necessity of poling this crystal contains a distribution of domains sized in the range of $1-8 \mu \mathrm{m},{ }^{14}$ providing an infinite set of grating vectors $\mathbf{g}$ for QPM of parametric processes. Thus the limitations of the bandwidth are mainly given by the transparency window of the crystal in the range of $0.4-6 \mu \mathrm{m} .{ }^{15}$ This is of special importance for ultrashort pulses, for which the full spectrum is converted with almost constant efficiency. ${ }^{16}$

The phase-matching conditions for SHG differ significantly when the SH photon is generated by two photons from the same pulse as in unidirectional frequency doubling (UDFD) $\left(\mathbf{k}_{2}=2 \mathbf{k}_{1}+\mathbf{g}\right)$ and when the fundamental photons originate from oppositely propagating pulses as in counterpropagating frequency doubling (CPFD) $\left(\mathbf{k}_{2}=\mathbf{k}_{1}-\mathbf{k}_{1}+\mathbf{g}^{\prime}\right)$. In both cases, the QPM in the crystal allows for SHG for all spectral components of the pulse in all spatial directions within a plane perpendicular to the crystal $z$ axis. For the practically important case of transverse SHG the relevant 


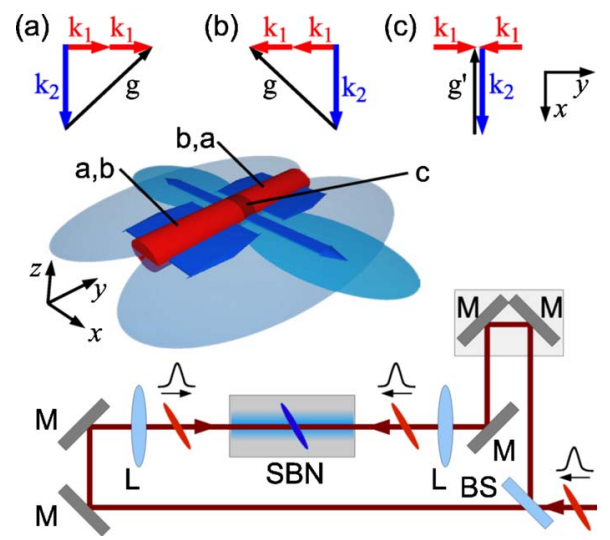

FIG. 1. (Color online) (Top row) Quasi-phase-matching conditions for transverse SHG: [(a) and (b)] unidirectional frequency doubling (UDFD) and (c) counterpropagating frequency doubling (CPFD). (Middle row) Schematic representation of the transverse $\mathrm{SH}$ emission of counterpropagating pulses: The SH of both UDFD and CPFD are emitted in the $X-Y$ plane. Arrows indicate the transverse direction of observation. (Bottom) Experimental setup: BS, beam splitter; M, mirror; L, cylindrical lens.

phase-matching conditions are depicted in Fig. 1 top). In the two regions [(a) and (b) in Fig. 1 (middle)] where the two pulses are propagating independently in opposite directions, the total SH signal is a sum of the signals generated by each UDFD process. This signal constitutes the background of the autocorrelation trace. In the region where the two counterpropagating pulses meet [(c) in Fig. 1 (middle)], the total SH signal is stronger because of the additional contribution from the CPFD process. The length of this region is proportional to the pulse duration. It can be clearly seen that for transverse SHG, different grating vectors $\mathbf{g}$ (and consequently different grating periods $d$ ) are required for UDFD [Fig. 1 (top), (a) and (b)] and CPFD [Fig. 1 (top), (c)] processes. While the grating period needed to phase match transverse CPFD is $2 d_{\mathrm{CPFD}}=2 \pi / \mathbf{g}^{\prime}=\lambda /\left(2 n_{\omega}\right)$, the UDFD requires period of $2 d_{\mathrm{UDFD}}=2 \pi / \mathbf{g} \approx \lambda /\left(2 n_{\omega} \sqrt{2}\right)$. This means that the phasematching period for the UDFD process is $\sqrt{2} / 2$ smaller than that of CPFD and, consequently, the former will involve $\sqrt{2} / 2$ smaller grating periods. In practice, such subwavelength size domains are not available in the crystal, ${ }^{14}$ thus the frequency conversion processes are phase matched via higher-order QPM processes. As UDFD and CPFD require different grating periods, one can expect a different efficiency for each of those processes depending on the availability of appropriate spectral components in the random domain structure. If one denotes the ratio of the efficiencies of both processes as $R=\eta_{\mathrm{UDFD}} / \eta_{\mathrm{CPFD}}$ then the signal to background ratio will be given by $P=2 / R+1$. For $R=1, P=3$ which is exactly the maximum value for the TPF process ${ }^{17} \mathrm{In}$ the typical case of SBN, however, the average domain size is $\sim 2.5 \mu \mathrm{m},{ }^{14}$ and consequently $R<1$. Thus one can expect an improved signal to background ratio $P>3$.

In the experiments, we use a femtosecond Ti:sapphire oscillator with pulse energies of $6 \mathrm{~nJ}$, a repetition rate of $76 \mathrm{MHz}$, and tunability in the range of $700-900 \mathrm{~nm}$. For the results presented in this letter the laser is set to $850 \mathrm{~nm}$. As shown in the schematic of the setup [Fig. 1 (bottom)], the output from the laser is split into two beams which are collinear and counterpropagating inside the $5 \mathrm{~mm}$ thick SBN crystal. In order to make use of the biggest component of the $\chi^{(2)}$ tensor $\left[d_{z z z}^{(2)}\right]$, both beams are extraordinarily polarized and propagate in the direction normal to the crystal's $z$ axis. Downloaded 04 Oct 2007 to 130.56 .65 .25 . Redistribution subject a)
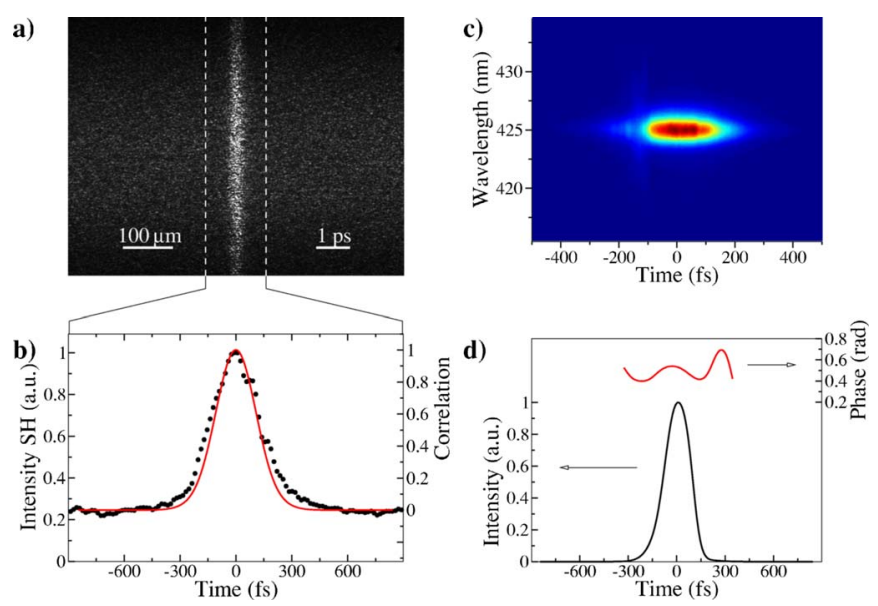

FIG. 2. (Color online) Comparison of the counterpropagating pulse based correlator with a GRENOUILLE. (a) Camera picture of transverse SH trace of the pulse. (b) Black dots, SH profile from area marked in (a); solid line, autocorrelation calculated from GRENOUILLE measurement. (c) GRENOUILLE trace. (d) Pulse and phase profiles retrieved from GRENOUILLE giving $T_{\mathrm{FWHM}}=189 \mathrm{fs}$.

The path lengths of both beams are set such that oppositely propagating pulses meet in the center of the crystal. Each incoming pulse train generates a second-harmonic trace in the crystal (via UDFD) that is recorded by a charge coupled device (CCD) camera mounted perpendicular to the beams and the crystal $z$ axis [Fig. 1 (middle)]. In the region where pulses overlap, the CPFD process leads to a clear peak on the SH background [Fig. 2(a)]. The emitted SH is extraordinarily polarized.

In the particular configuration, when the two counterpropagating signals are identical, the generated normalized signal $I_{\mathrm{tot}}(\tau)$ is given as $I_{\mathrm{tot}}(\tau)=1+(2 / R) G^{(2)}(\tau)$, where $G^{(2)}(\tau)=\langle I(t) I(t+\tau)\rangle /\left\langle I^{2}(t)\right\rangle$ represents exactly the autocorrelation of the pulse. This experimental geometry results in a mapping of time $(\tau)$ into a space (longitudinal) coordinate $(s)$ via the simple relation $\tau=s 2 n_{\omega} / c$, where $n_{\omega}$ is the SBN refractive index dependent on the optical frequency, ${ }^{18}$ and $c$ is the speed of light in vacuum. Hence, the longitudinal distribution of the SH signal represents a timedependent autocorrelation signal. We note that this is a single-shot technique, and it does not require any variable differential delay during the correlation recording. Measurements with an uncooled CCD camera were done at power levels down to $0.26 \mathrm{MW} / \mathrm{cm}^{2}$, four orders of magnitude lower than those usually used with TPF. This significantly lower power requirement is mainly due to the confinement of the emitted $\mathrm{SH}$ in a plane compared to the emission in the full solid angle from the TPF process.

The measurement accuracy is primarily limited by the imaging of the correlation trace onto the camera. For recording, we use an optical microscope at 4.5 magnification. To avoid an image distortion caused by the limited depth of field, the beams are focused in the crystal by cylindrical lenses $(f=50 \mathrm{~mm})$, resulting in a beam width of $33 \mu \mathrm{m}$ along the observation direction. Additionally, we measured the pulse duration with a GRENOUILLE [Figs. 2(c) and 2(d)], obtaining a reference measurement [Fig. 2(b), red solid line] for comparison with our SH autocorrelation trace (black dots). In a series of measurements we find the two methods to disagree by no more than $10 \%$, including the dispersion of the $190 \mathrm{fs}$ pulse in $2.5 \mathrm{~mm}$ of SBN, that accounted for apthe $190 \mathrm{fs}$ pulse in $2.5 \mathrm{~mm}$ of $\mathrm{SBN}$, that accounted for ap-
AlP license or copyright, see http://apl.aip.org/apl/copyright.jsp 
a)

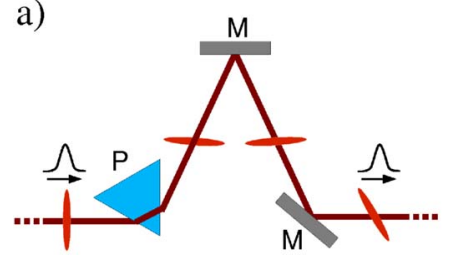

d)

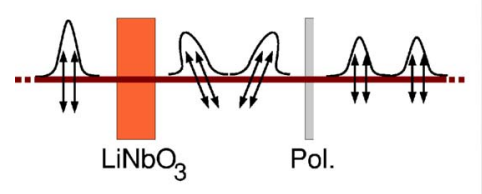

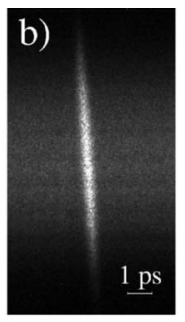

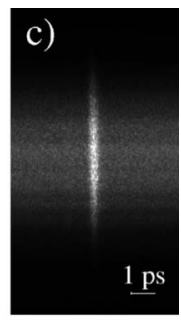

e)

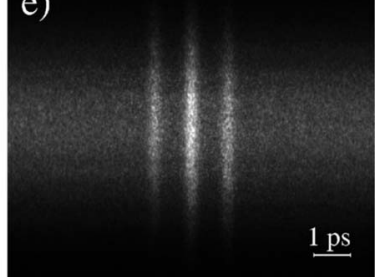

FIG. 3. (Color online) Detection of pulse front tilt and multipulse structures. (a) Optical system used to induce a pulse front tilt. [(b) and (c)] Autocorrelation traces of the tilted and straight front pulses. (d) Schematic of the generation of a pulse doublet by means of a birefringent crystal. (e) Autocorrelation trace of a doublet for 989 fs delay between the two pulses.

proximately $4.5 \%$ of the error. The effect of dispersion can be easily reduced by using shorter crystals. We note that the signal to background ratio $P$ in this measurement is approximately 4 .

Apart from the pulse duration measurements, the correlator in Fig. 1 (bottom) can also be used to visualize unambiguously the tilt of the front of an optical pulse. Such a pulse front tilt (PFT) (which is typically caused by dispersive elements such as prisms, gratings, or wedges) leads to an effective longer pulse duration and hence a lower peak power in the focal plane of the beam. By placing a $60^{\circ}$ (SF11 Schott glass) prism into the beam path [Fig. 3(a)], a PFT was introduced that can be clearly seen in the $4^{\circ}$ tilt of the correlation trace [Fig. 3(b), compare with Fig. 3(c)].

The large time window of our technique $(1 \mathrm{~mm} \mathrm{SBN}$ is sufficient to monitor $7 \mathrm{ps}$ ) allows for monitoring more complex temporal structures consisting of multiple pulses. To demonstrate this feature, a pulse doublet is generated by passing the beam through a thin $(3.2 \mathrm{~mm})$ birefringent (lithium niobate) crystal followed by a polarizer, as depicted in Fig. 3(d). The ordinarily polarized component of every pulse gets delayed by roughly 1 ps with respect to its extraordinarily polarized counterpart. The polarizer combines these two components to a pulse doublet. The autocorrelation trace shown in Fig. 3(e) clearly resolves the two components of the doublet and allows for the delay between them to be precisely measured. For comparison, in the analogous experiment, GRENOUILLE averages over the two pulses due to its narrow time window, hence the information about the double structure is lost.

Even more information about a pulse signal can be gained if one of the counterpropagating beams is a pulse train with well defined properties, e.g., generated by an optical clock. In that case, the SH signal represents a cross correlation of the reference and signal pulses. Due to the ability of the random ferroelectric domain structure to phase

match parametric processes over an ultrabroad frequency range, the reference signal does not need to have the same carrier frequency as the measured signal. To the contrary, different carrier frequencies of both pulse trains allow for the two background SH signals to be filtered out from the sumfrequency correlation signal, resulting in a background-free correlation measurement. Furthermore, background-free autocorrelation can be achieved when the counter propagating beams are directed along the crystal $z$ axis. In this case only the process of CPFD gives rise to a plane emission but at a lower efficiency due to the lower value of the governing

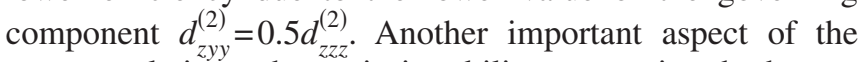
crosscorrelation scheme is its ability to monitor both synchronization and timing of a pulse train. Even a small desynchronization between the reference and signal source will lead to a shift of the correlation peak which can be easily measured. On the other hand, a pulse jitter will result in broadening and flattening of the correlation trace.

In conclusion, we demonstrated a solid-state pulse correlation scheme based on transverse phase-matched parametric upconversion in disordered nonlinear optical media. While the correlation technique is not sensitive to the complex pulse shape, it can be used to monitor pulse properties such as duration and front tilt as well as to measure timing and synchronization in pulse trains. We discussed the corresponding phase-matching conditions for the counterpropagating beams in this random ferroelectric domain structure that allows for an operation over an ultrabroad frequency range and an improved signal to background ratio of the correlation trace. Its simplicity and the possibility of a compact integration with standard fiber components make this technique attractive for a wide range of applications.

The authors acknowledge the support of the Australian Research Council.

${ }^{1}$ H. P. Weber, J. Appl. Phys. 38, 2231 (1967).

${ }^{2}$ D. J. Kane and R. Trebino, Opt. Lett. 18, 823 (1993).

${ }^{3}$ C. Iaconis and I. A. Walmsley, Opt. Lett. 23, 792 (1998).

${ }^{4}$ P. O'Shea, M. Kimmel, X. Gu, and R. Trebino, Opt. Lett. 26, 932 (2001).

${ }^{5}$ D. J. Bradley and G. H. C. New, Proc. IEEE 62, 313 (1974).

${ }^{6}$ J. A. Giordmaine, P. M. Rentzepis, S. L. Shapiro, and K. W. Wecht, Appl. Phys. Lett. 11, 216 (1967).

${ }^{7}$ F. Theberge, S. M. Sharifi, S. L. Chin, and H. Schröder, Opt. Express 14, 10125 (2006).

${ }^{8}$ R. Normandin and G. I. Stegeman, Opt. Lett. 4, 58 (1979).

${ }^{9}$ R. Normandin and G. I. Stegeman, Appl. Phys. Lett. 36, 253 (1979).

${ }^{10}$ R. Normandin, R. L. Williams, and F. Chatenoud, Electron. Lett. 26, 2088 (1990).

${ }^{11}$ G. D. Landry and T. A. Maldonado, J. Opt. Soc. Am. B 21, 1509 (2004).

${ }^{12}$ N. D. Whitbread, J. A. R. Williams, J. S. Roberts, I. Bennion, and P. N. Robson, Opt. Lett. 19, 2089 (1994).

${ }^{13}$ A. Otomo, G. I. Stegeman, M. C. Flipse, M. B. J. Diemeer, W. H. G. Horsthuis, and G. R. Mohlmann, J. Opt. Soc. Am. B 15, 7759 (1998).

${ }^{14}$ J. J. Romero, C. Aragó, J. A. Gonzalo, D. Jaque, and J. García Solé, J. Appl. Phys. 93, 3111 (2003).

${ }^{15}$ M. Horowitz, A. Bekker, and B. Fischer, Appl. Phys. Lett. 62, 2619 (1993).

${ }^{16}$ R. Fischer, S. M. Saltiel, D. N. Neshev, W. Krolikowski, and Yu. S. Kivshar, Appl. Phys. Lett. 89, 191105 (2006).

${ }^{17}$ J. H. Bechtel and W. Smith, J. Appl. Phys. 46, 5055 (1975).

${ }^{18}$ Th. Woike, Phys. Status Solidi A 186, R13 (2001). 